\title{
Ultrastructural Diagnosis of Tumors
}

\author{
J. A. Tucker*
}

*Department of Pathology, University of South Alabama, 2451 Fillingim Street, Mobile, AL 36617

Electron microscopy has been widely used in the field of medicine. The technique has served to expand the understanding of disease, and in some cases, electron microscopy has actually defined disease categories. During the last forty years, electron microscopy has been used not only as a research tool in medicine, but it became a standard diagnostic method for pathologists.

Specifically in the area of tumor diagnosis, electron microscopy proved very important. Though most cases were diagnostic by light microscopy alone, some were not. Ultrastructural features such as desmosomes, secretory granules, tonofilament bundles, lakes of glycogen, and premelanosomes proved useful in the accurate diagnosis of tumors. At its peak, ultrastructural examination in surgical pathology proved to be of diagnostic importance in about $8 \%$ of tumor cases.

With the widespread application of immunohistochemistry in the 1980's and with the addition of other molecular techniques, the number of tumor cases requiring ultrastructural examination has significantly diminished. For example, prior to immunohistochemistry, simply distinguishing whether an ulcerated skin tumor was a malignant melanoma or a squamous cell carcinoma could be difficult or impossible by light microscopy, and ultrastructural examination was crucial in such cases. Today, those formerly challenging cases can often be easily diagnosed using common, standard immunohistochemical stains.

While the role of ultrastructural diagnosis of tumors has diminished, the technique remains an essential tool in a subset of human tumors. Tumors exhibit immunohistochemical/molecular heterogeneity, and the results of such studies may be inconclusive or yield contradictory information. In some instances, electron microscopy may still yield a definitive diagnosis while other methods do not. And in other cases, while electron microscopy alone may not prove diagnostic, the consideration of the ultrastructural findings along with all other findings may help to refine or extend a diagnosis, which may, in turn, help guide treatment.

In the diagnostic evaluation of tumors, the advances in other techniques have significantly diminished the percentage of cases requiring ultrastructural examination. In a small subset of cases, though, electron microscopy continues to provide clinically useful information in the evaluation of tumors. 


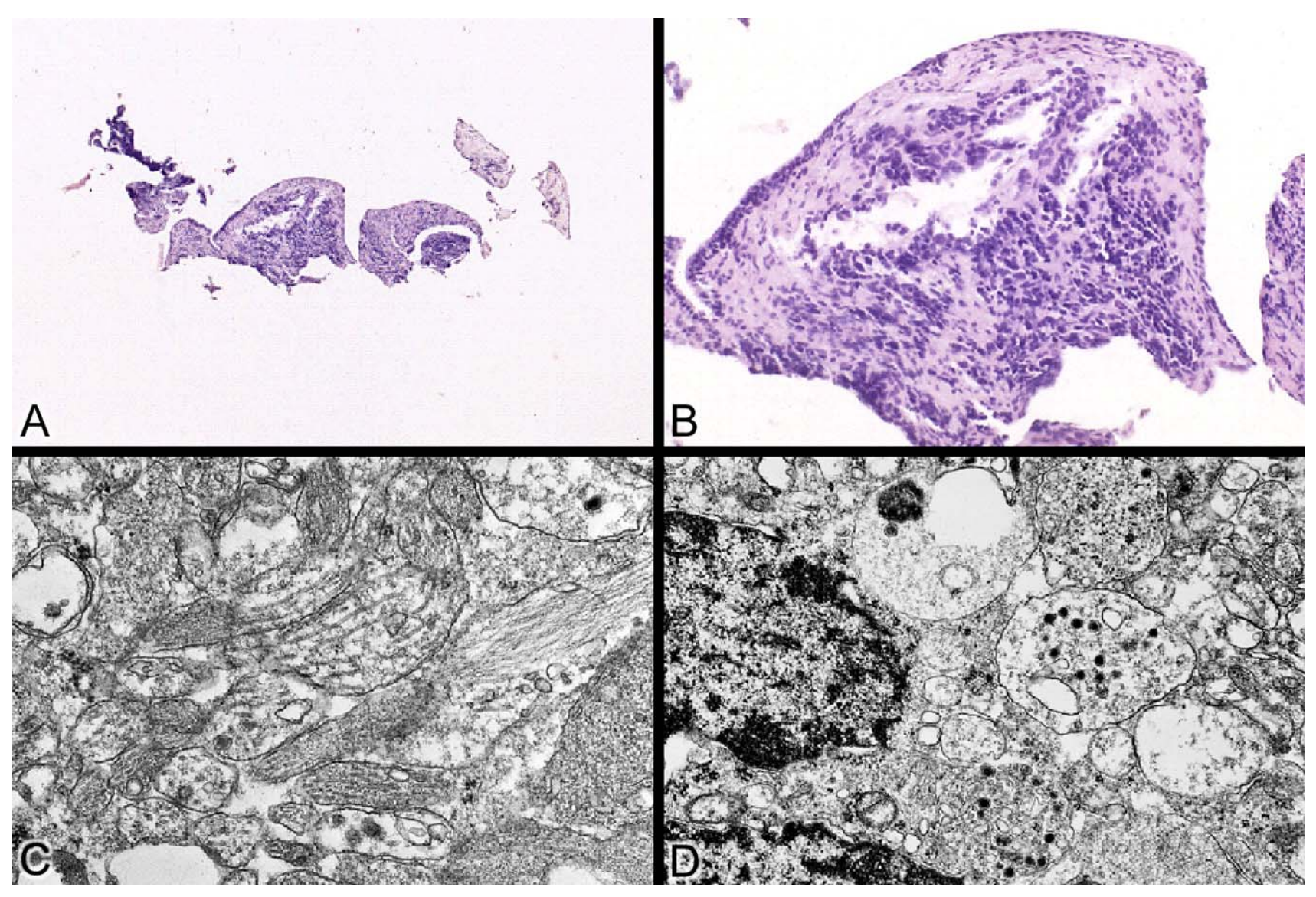

Figure 1 Example of contemporary role of electron microscopy in tumor diagnosis. A 2 year old boy presented with a mass involving the right ear and mastoid. A. A small biopsy was obtained. B. The tumor consisted of anaplastic cells. Lymphoma was suspected; immunohistochemical stains were negative. C. Ultrastructural examination revealed that the tumor cells had numerous cell processes with microtubules. D. Neurosecretory granules were also present. While the light microscopic findings and immunohistochemical studies proved inconclusive, ultrastructural examination was diagnostic of neuroblastoma. 